\title{
Flexible Iterative Learning Control Based Expert System and Its Application
}

\author{
Liu Zuojun, Liu Zhihu, Zu Linan and Yang Peng
}

\author{
Department of Automation, Hebei University of Technology, Tianjin, PR China 300130
}

\begin{abstract}
A scheme of expert system based on iterative learning control is proposed. Iterative learning control can obtain control experience from the historical data to build the knowledge base of expert system. Considering some uncertain factors, a flexible measure is adopted in iterative learning control (ILC). Simulation proves the feasibility and effect of the air conditioning control expert system based on flexible iterative learning control (F-ILC). Finally, a feedback compensation unit is incorporated against irregular heavy disturbance.
\end{abstract}

Key Words : Expert System, Iterative Learning Control, Flexible

\section{Introduction}

Expert system is an artificial intelligence application that uses a knowledge base of human expertise for problem solving. Its success is based on the quality of the data and rules obtained from the human expert ${ }^{[1]}$. It derives its answers by running the knowledge base through an inference engine, which is software that interacts with the user and processes the results from the rules and data in the knowledge base. There are many successful examples of expert system in medical diagnosis, equipment repair, investment analysis, financial, estate and insurance planning, vehicle routing, contract bidding, production control and training ${ }^{[1-3]}$. In practice, expert systems perform both below and above that of a human, which is mainly due to the quality of human expert knowledge summary. Iterative learning control (ILC) ${ }^{[7-9]}$ refers to the problem of finding an appropriate control input that causes the output to track a prescribed trajectory defined in a finite time interval by iteration trials without knowing the accurate plant model. Learning control applies naturally in processes where the same task is repeated many times, and the system is designed to return to the same initial conditions before commencing the next cycle or trial. For example, the machine resets automatically to a home position as one finished work piece moves out before the next work piece arrives. Robots performing conveying, assembling, welding and spray-painting all have the repetitive nature. Besides electro-mechanical systems, application of ILC can also extend to batch process system, such as the rapid thermal processing, batch reactors, and other batch chemical processes.

Standard ILC can only be used in processes where the same desired parameters are repeated turn by turn, which means there should be no difference in each turn. However, in many systems with repetitive nature, the inevitable uncertain

Manuscript received Jun. 14. 2009; revised Sep. 6. 2009.

This work is supported by fundamental research plan of Tianjin Municipality (07JCYBJC05300) in China. Also, the aid from lab of intelligent robotics in Nanyang Technological University is appreciated. disturbances limit the application of ILC. So a Flexible ILC scheme is proposed to extent the application field of ILC. And in this paper, F-ILC is used for building knowledge base of expert system for the control systems with repetitive nature.

The rest of the paper is organized as follows. Section 2 reviews standard ILC and presents the scheme of flexible ILC. Section 3 introduces the general conception of expert system. Section 4 presents the simulation results of expert system based on F-ILC used in the application of air conditioning control. Section 5 provides concluding remarks.

\section{Flexible ILC}

\subsection{ILC}

Iterative learning control uses the historical control date in the past working turns. Like human learning experiences in repetitive trails, ILC obtains higher control precision turn by turn. And the error will decrease in the process of iterative learning. As shown in Fig.1, given a desired output trajectory $y_{d}(t)$ for an operation period $[0, \mathrm{~T}]$, a control input $u(t)$ is made out through iterative learning to keep the system output $y(t)$ tracing $y_{d}(t) .{ }^{[6-9]}$

ILC obtains $u(t)$ in the way of iterative learning function, in which a correction learning function makes out a function array $\left\{u_{k}(t)\right\}$ from each repetitive turns, shown as below.

$$
\begin{gathered}
u_{k+1}(t)=u_{k}(t)+U\left(e_{k}(t), t\right) \\
e_{k}(t)=y_{d}(t)-y_{k}(t)
\end{gathered}
$$

where $k=0,1,2, \ldots$ is iterative turns; $y_{k}(t)$ is output of the system; $u_{k}(t)$ is the output of ILC controller. $u_{k}(t)$ obtains the control experiences from the former repetitive turns. And $U\left(e_{k}(t), t\right)$ includes the error correction information in the $k$-th turn. $u_{k+l}(t)$ is so obtaining optimum turn by turn.

Most existing ILCs are of either D-type, P-type, I-type or their variations ${ }^{[7-9]}$, described as follows.

$$
u_{k+1}(t)=u_{k}(t)+K_{P} e_{k}(t)+K_{I} \int_{0}^{t} e_{k}(t) d t+K_{D} e_{k}^{\prime}(t)
$$

Where $K_{P}, K_{I}$ and $K_{D}$ are constant gain matrixes. 


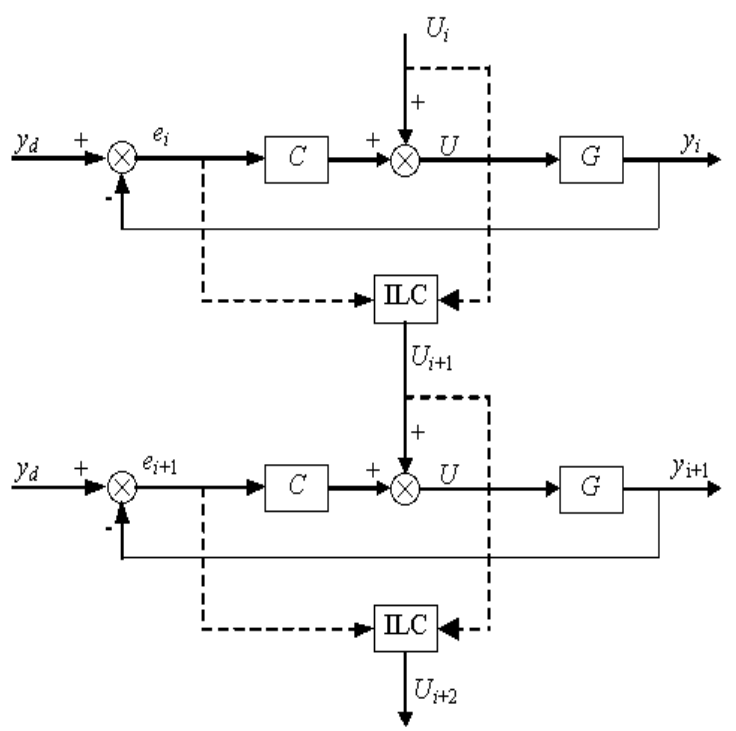

Fig. 1. The iterative learning control

\subsection{F-ILC Scheme}

Flexibility is the ability of a control system to adapt the change in and out of the system. However, standard ILC applies in processes where the same desired parameters are repeated turn by turn, which means there should be no difference in each turn. Although there are repetitive natures in many control systems, the inevitable uncertain disturbances limit the application of ILC. For example, there is regular nature in the repetitive working process day by day in air conditioning system, but it is still not fit for the application of ILC because of some irregular factors, such as the outer temperature, the human flux distribution. The same issue also exists in the control of lower prosthesis, where the steps are basically same turn by turn with slim difference. So the key point is how to make the ILC more flexible to be used in these control system with acceptable error tolerance.

So a F-ILC scheme is proposed below. At the beginning of iterative learning, PD-type ILC is used. The D factor may improve the learning rate and make the error convergent rapidly.

$$
u_{k+1}(t)=u_{k}(t)+K_{P} e_{k}(t)+K_{D} e_{k}^{\prime}(t)
$$

Due to the irregular factor of AC system in each turn, the working processes are not strictly same in every repetitive turn. When the error reduces small, as shown in the equation below, the root mean square (rms) error in each day converges into a threshold value

$$
|| e_{k}(t) \|<\left|e_{a}\right|
$$

The D-type ILC will cause the error divergent even the disturbance is small in magnitude. So if Eq.(6) is met, D factor would not be used any more, and only P-type ILC is used for stable convergence.

$$
u_{k+1}(t)=u_{k}(t)+K_{P} e_{k}(t)
$$

Standard ILC applies only in processes where the same desired parameters are repeated, including the start and end point, as well as all the working point in every cycle or turn. While some systems do not satisfy these conditions, so when the error converge into another much smaller threshold value, which may also be set though simulation or experiment, as below.

$$
\left\|e_{k}(t)\right\|<\left|e_{b}\right|
$$

The system is basically stable and the error is very small and acceptable. The control precision has met with the tolerance of the system, so a control dead-zone is set to ILC. No more iterative learning is necessary, or there may be error divergence. The control experience data may be stored in a knowledge base of expert system. The knowledge base is created according to various typical sorts of working conditions. After the knowledge base is finished, the controller may obtain the corresponding control experience according to the present background conditions sort of the system.

As the ILC presented above could be used in the systems not strictly same in every cycle or turn, and it may permit small scope error, so it is named flexible ILC, F-ILC.

\section{Expert System}

An expert system is a computer program that simulates the judgment and behavior of a human or an organization that has expert knowledge and experience in a particular field. Typically, such a system contains a knowledge base containing accumulated experience and a set of rules for applying the knowledge base to each particular situation that is described to the program. A set of "if-then rules" are used to look up corresponding control experience in the knowledge base according to the practical working conditions.

Generally, the performing effect of expert systems is mainly determined by the quality of human expert knowledge summary. However, generally, the human experience is not assured as the optima ones, and sometimes it might be improper and low efficient operating experience. Moreover, the process of building a knowledge base usually needs a long period, in which human expert operating skill is accumulated, extracted and summarized as a set of "if-then rules". So the expert system is not an easy making thing, which greatly limits its application field.

In practice, there are many systems working with repetitive and periodic nature. So iterative learning might be used for obtaining the knowledge in an automatic way that can be realized by computer program. As a result, it may make the expert system easy to realize. Also, the improper knowledge and tough process of knowledge accumulation and extraction can be avoided. 


\section{Application of Expert System Based on F-ILC}

\subsection{Control Plant Analysis}

Air conditioning system is usually controlled by means of PID control or Bang-bang control, which are all based on feedback ${ }^{[11-13]}$. Due to the inherit delay and lag of regulation process and actuators, the large range fluctuation and long time transient process of the regulation are inevitable, and accordingly the control effect and energy efficiency are not satisfied.

In many cases, there is some kind of regularity in the working process of air conditioner in each day. That is because the load of AC is mainly determined by two factors, the outdoor temperature and the indoor activities of people. And the outdoor temperature runs similar curve each day of the same whether. The temperature is always lower in the early morning, and runs higher till the noon, then falls little in the dusk. Also, there is similar curve in the indoor activities of people each day. For example, the activities of passengers and staffs with their office electrical apparatuses in airport and railway station are associated with the schedules of planes and trains, so the AC load curves caused by it are similar. There are regularities in some other places too, such as hospital, supermarket and cinema. According to the praxeology, the activity of single people is random and disordered; while the activities of swarm people are self-organized and obey some kinds of statistics regularity. Human flux distribution of 4 common sunny workdays in a supermarket is statistically compared in Fig.2. The general statistics regularity does exist to some extend.

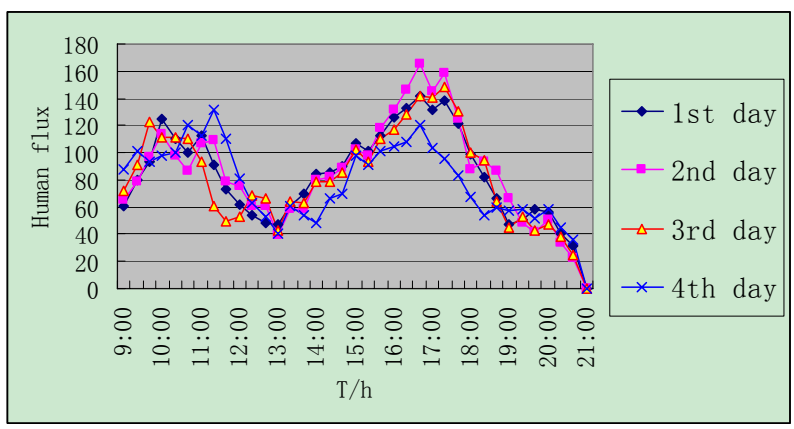

Fig. 2. The statistics regularity of human flux

As there is regularity in the working process of AC system in each day, so the historical control data can provide experience for the control in next day, as well as for the building of knowledge base in expert system.

However, there still are some irregularities in each day. As it is comparative small to the whole $\mathrm{AC}$ load, it may be considered as disturbance to be eliminated by feedback control.

Take a supermarket as an example ${ }^{[11]}$. The regularity nature of AC system makes the energy consumption curve in each day similar, as shown in Fig.3. On one hand, the curves are similar due to the regularity of outdoor temperature and indoor activities of people. On the other hand, there are some differences caused by random factors of indoor activities and outdoor whether. As stated above, the standard ILC does not fit for such kind of systems, while the F-ILC may be used. In Fig.3, the 8 days load curves are chose under same whether and indoor activity conditions. The days in this ILC sample set are all sunny and fuzzy hot whether weekdays. That is to say, the rainy days or weekend days are in other ILC sample sets. And an expert system may be build based on F-ILC.
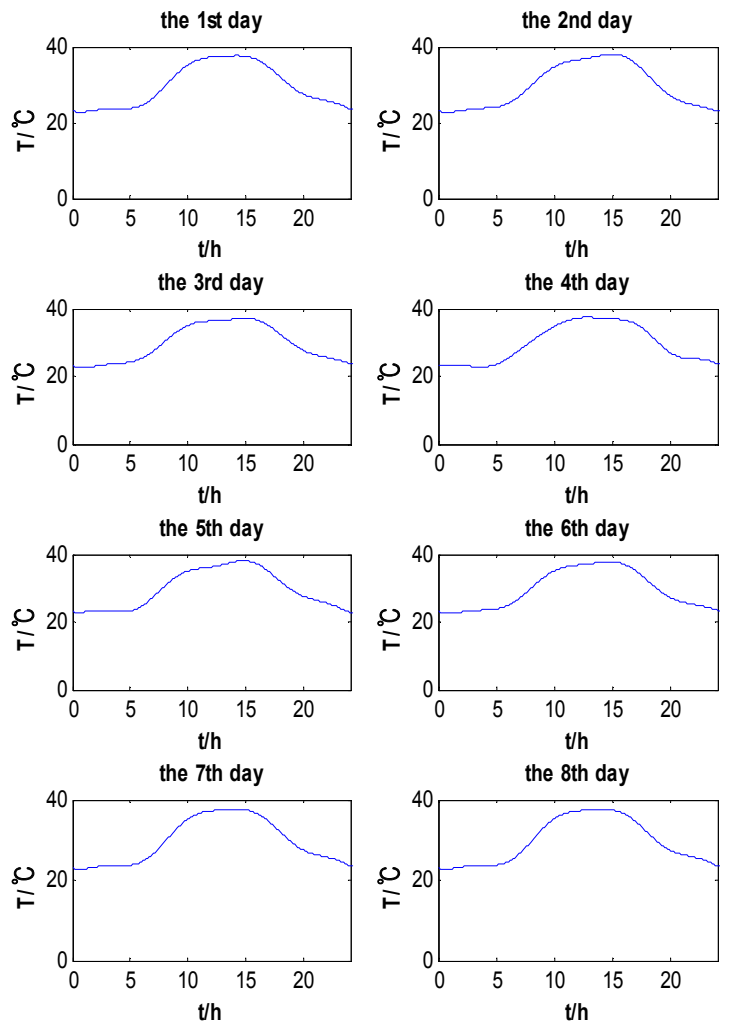

Fig. 3. The energy consumption of air conditioning

\subsection{Solution Scheme}

First of all, divide the working condition of the AC system into several fuzzy sets according to weather forecast, which may include strong hot, hot, weak hot, comfortable, cool, and so on. Next, each fuzzy set is divided into secondary subsets according to the human flux activities, which may include working days, weekend days, big sales days and so on. In another words, it can be processed as a Cartesian product between the weather fuzzy set and human flux category set. Then F-ILC is utilized for each fuzzy subset, and different control knowledge would be obtained to fill out knowledge base of expert system for all kinds of $\mathrm{AC}$ working background conditions.

Fig. 3 shows 8 days AC load curves in the same fuzzy subset. All the curves are generally same, but there are still some small differences with each other days. And F-ILC might be fit for this kind of plant to draw knowledge from it in iterative learning way.

After the knowledge base of expert system is finished, the 
control system might be put into practice. The inference engine derives conclusion from the facts and rules contained in the knowledge base using the condition matching technique. And the knowledge obtained in F-ILC is called by means of "if... then" rules according to the AC working fuzzy subset information via the user interface of expert system.

To sum up, the structure of flexible ILC based expert control system is shown as below.

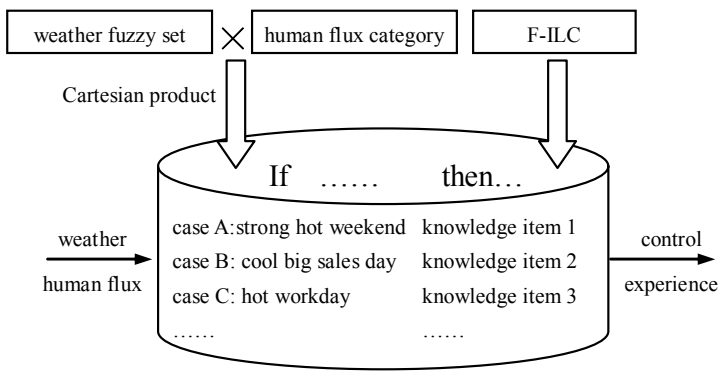

a) Structure of knowlede base

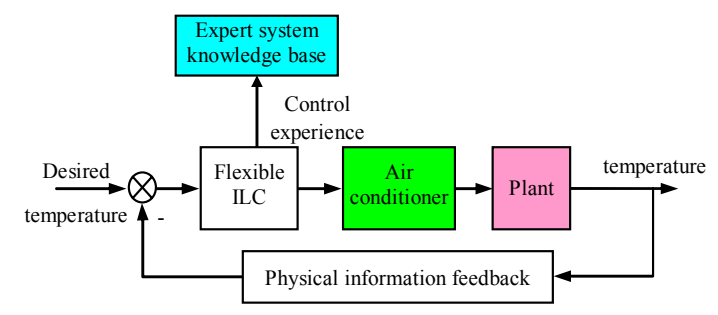

b) The building of knowledge base

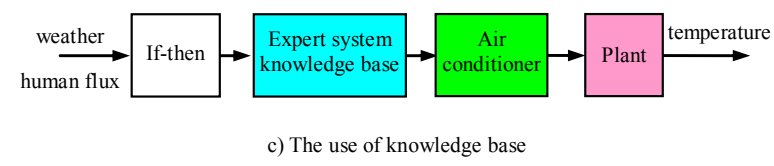

Fig. 4. Structure of flexible ILC based expert control system

\subsection{Control Effect}

The input of the AC control system is the error between the desired air temperature and return air temperature. And the output of system is the cooling capacity, which is temperature error between supply and return air multiplied by the air volume. The simulation is run in Matlab. The indoor temperature of AC F-ILC system is shown in Fig.5, in which the control precision is improved day by day based on F-ILC. With the decrease of error, there is no divergence trend which will occur in standard ILC. The error convergence is shown in Fig.6, which demonstrates the decrease of error rms of each day turn by turn. As the F-ILC archives high control precision in AC system, it could obtain better energy saving effect. So the final control parameters can be stored into the knowledge base of expert system.

After the whole knowledge base is finished, each piece of knowledge obtained from F-ILC might be active according to the present weather and human flux conditions information via the user interface of expert system. And good control effects might be obtained.
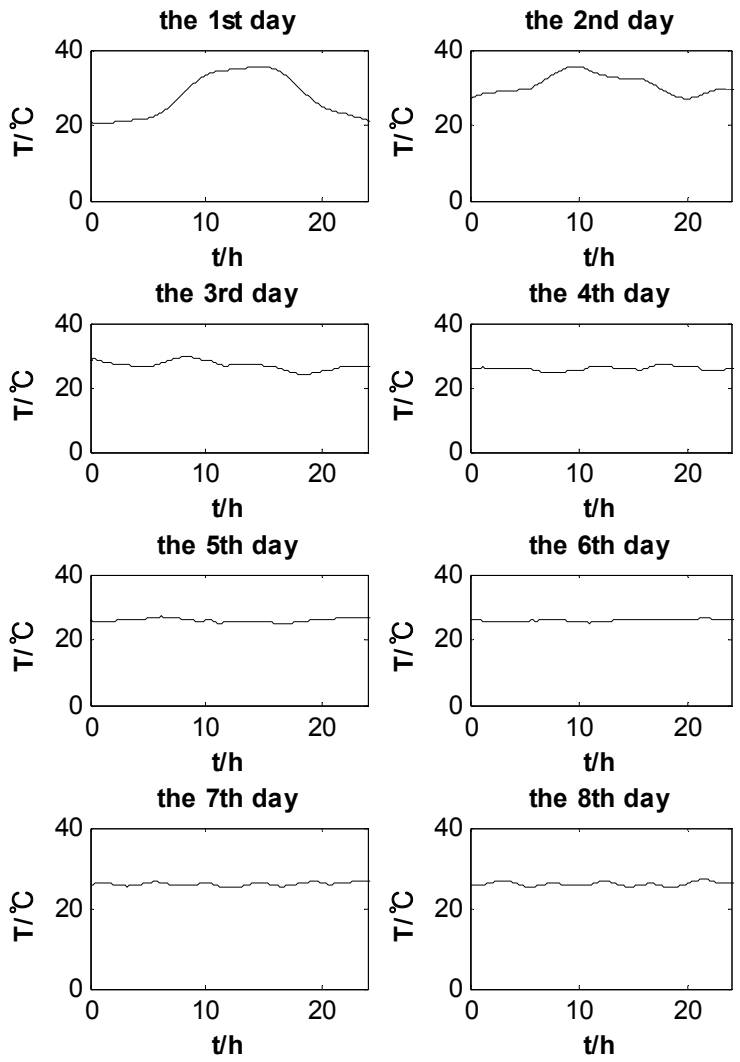

Fig. 5. The curve of energy saving

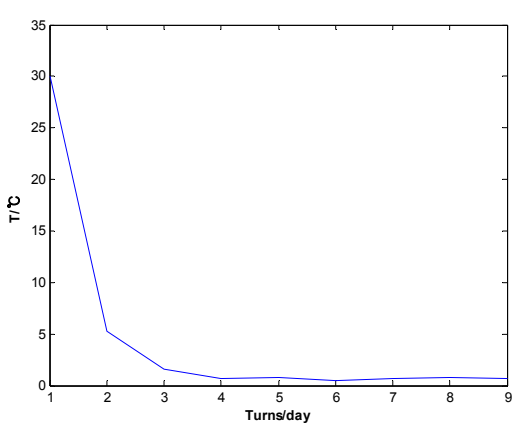

Fig. 6. Error curve of F-ILC

\subsection{Feedback Compensation against Irregular Disturbance}

Although the load curves of AC system of each day with same weather and human flux condition are usually similar, some irregular heavy disturbances are inevitable. For example, maybe some day, many teenagers in a nearby school flow into the supermarket because they are all requested to buy a marker by their teachers. As the expert control system proposed in this paper is a kind of open loop control, it could not eliminate the error caused by the irregular heavy disturbances and poor control effect might occur. As a result, pure F-ILC based expert system is not enough for ideal control. Then a closed loop control unit based on real time feedback is incorporated as an essential compensation measure.

The feedback compensation unit, a PID controller, will be active when the real time error $e_{i}(t)$ exceeds a predetermined 
threshold value $e_{c}$ as below.

$$
\begin{gathered}
\left|e_{i}(t)\right|>\left|e_{c}\right| \\
u_{f b c}(t)=K_{P} e_{i}(t)+K_{I} \int_{0}^{t} e_{i} k(\tau) d \tau+K_{D} e_{i}^{\prime}(t)
\end{gathered}
$$

The overall control output is composed of both the feedback part and the expert system part. So the irregular heavy disturbance can be eliminated and ideal control effect is obtained. The simulation is shown in Fig.7, in which the dashed line, denoted by $y 1$, is pure expert control system, while the solid line, denoted by $y 2$, is the ones with feedback compensation.

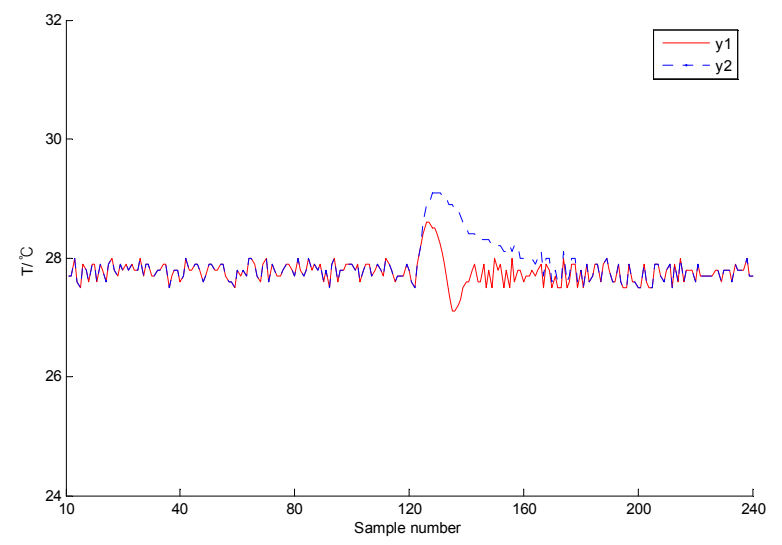

Fig. 7. Feedback compensation against irregular disturbance

\section{Conclusion}

A flexible ILC is proposed to build the knowledge base of expert system for the system with similar repetitive nature. It expends the application field of ILC. As F-ILC may learn the control experience to build the knowledge base in an automatic way, so it avoids the tough process of knowledge summary from experienced operators, as well as some possible wrong knowledge in the brain of operators. Furthermore, a closed loop control unit based on real time feedback is incorporated with expert control system as an essential compensation measure against irregular heavy disturbance.

\section{References}

[1] Tang Jianzhong, Wang Qingfeng, Bi Zhiyue. "Expert system for operation optimization and control of cutter suction dredger". Expert Systems with Applications. vol. 34, no. 3, pp. 180-2192, 2008.

[2] Reaz, M.B.I.; Choong, F.; Sulaiman, M.S.; Mohd-Yasin, F.; Kamada, M.; "Expert system for power quality disturbance classifier", IEEE Transactions on Power Delivery. vol. 22, no. 3, pp. 1979-1988, 2007.
[3] Men-Shen Tsai; "Development of an object-oriented service restoration expert system with load variations" IEEE Transactions on Power Systems, vol. 23, no. 1, pp. 219-225, 2008.

[4] Leduc, R.J.; Lawford, M.; Pengcheng Dai; "Hierarchical interface-based supervisory control of a flexible manufacturing system”, IEEE Transactions on Control Systems Technology, vol. 14, no. 4, pp. 654-668, 2006.

[5] Qing-lei Hu; Zidong Wang; Huijun Gao; "Sliding mode and shaped input vibration control of flexible systems". IEEE Transactions on Aerospace and Electronic Systems. vol. 44, no. 2, pp. 503-519, 2008.

[6] Mingxuan Sun, Danwei Wang. "Iterative learning control design for uncertain dynamic systems with delayed states", Dynamics and Control. vol. 10, pp. 341-357, 2000.

[7] R. W. Longman. Iterative learning control and repetitive control for engineering practice, International Journal of Control, vol. 73, no. 10, pp. 930-954, 2000.

[8] HU Yue, ZHAI Chunyan. "Current status and expectation of iterative learning control", Process Automation Instrumentation. vol. 26. no. 6, pp. 1-4, 2005

[9] Piao Fengxian, Zhang Qingling, Wang Zhefeng. "Analysis of convergence rate for iterative learning control", Journal of Northe Astern University(Natural Science). vol. 27, no. 8, pp. 835-839, 2006

[10]Zuojun Liu, Junrui Zhang, Sufang Pang, Peng Yang. "The research and application of control method based on flexible ILC'. Proc. of IEEE WCICA 2008. Chongqing: pp. 5744-5749, 2008.

[11] Xu Wangfa, Zhan Xu, "Dynamic simulation of energy consumption for large scale supermarkets", Heating Ventilating \& Air Conditioning. vol. 36, no. 11, pp. 106-109, 2006.

[12] Aucevic M. Energy "Efficient office building design". ASHRAE Journal, vol. 45, no. 1, pp. 26-29, 2005.

[13] Liu Z., Qi W. "System integration control of HVAC in intelligent building". Proc. of ICMLC2004. Shanghai: vol. 2, pp. 1125-1128, 2004.

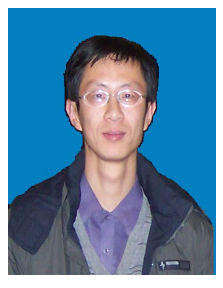

\section{Liu Zuojun}

He received his B.S. and M.S. degree in the Department of Automation from Hebei University of Technology, Tianjin, China, in 1994 and in 2000 respectively, and the Ph.d degree in Department of Automation from Nankai University, Tianjin, China, in 2005. $\mathrm{He}$ is now the head of Department of Automation in Hebei University of Technology. His research interest includes intelligent robotics and intelligent building. 


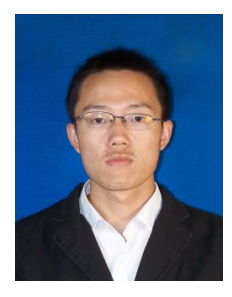

Liu Zhihu

He received his B.S. degree in the Department of Automation from North China Institute of Science and Technology, Langfang, China, in 2008. He is now a master student in Hebei University of Technology

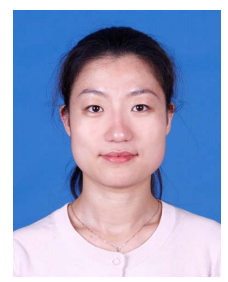

\section{Zu Linan}

She received his B.S., M.S. and Ph.d degree in the Department of Automation from Jilin University, Changchun, China, in 1999, in 2002 and in 2006 respectively. She is now associated professor of Department of Automation in Hebei University of Technology. Her research interest includes intelligent robotics and control theory.

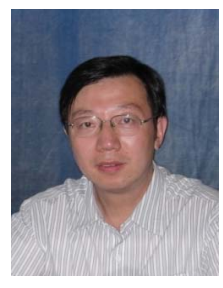

\section{Yang Peng}

He received his B.S. and Ph.d. degree in the Department of Automation from Hebei University of Technology, Tianjin, China, in 1982 and in 1998 respectively. He is now a professor of Department of Automation in Hebei University of Technology. His research interest includes intelligent robotics and intelligent control theory and application. 